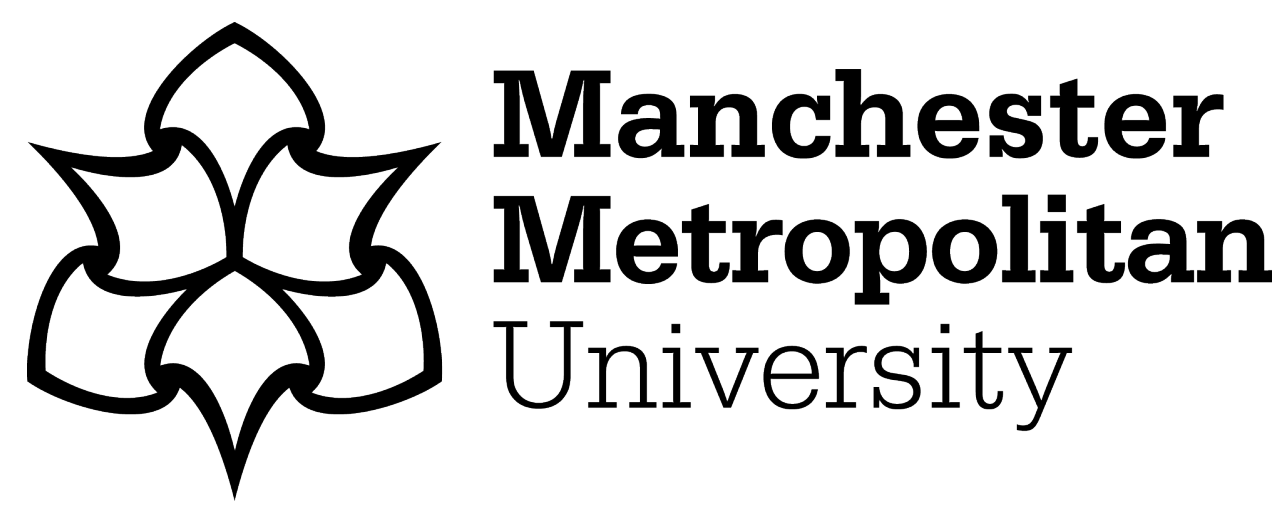

Marshall, Ben ORCID logoORCID: https://orcid.org/0000-0003-2557-5399, Wright, David ORCID logoORCID: https://orcid.org/0000-0001-95680237, Holmes, Paul ORCID logoORCID: https://orcid.org/0000-00030821-3580, Williams, Jacqueline and Wood, Greg ORCID logoORCID: https://orcid.org/0000-0003-0851-7090 (2020) Combined action observation and motor imagery facilitates visuomotor adaptation in children with Developmental Coordination Disorder. Research in Developmental Disabilities, 98. p. 103570. ISSN 0891-4222

Downloaded from: https://e-space.mmu.ac.uk/624674/

Version: Accepted Version

Publisher: Elsevier

DOI: https://doi.org/10.1016/j.ridd.2019.103570

Usage rights: Creative Commons: Attribution-Noncommercial-No Derivative Works 4.0

Please cite the published version 
5

\section{Combined action observation and motor imagery facilitates visuomotor adaptation in children with Developmental Coordination Disorder} 7

1. Research Centre for Musculoskeletal Science and Sports Medicine, Department of Sport and Exercise Sciences, Faculty of Science and Engineering, Manchester Metropolitan University, Manchester, UK

2. Research Centre for Musculoskeletal Science and Sports Medicine, Department of Psychology, Faculty of Health, Psychology and Social Care, Manchester Metropolitan University, Manchester, UK

3. Institute for Sport and Health, College of Sport and Exercise Science, Victoria University, Melbourne, Australia

*Corresponding author information: Dr Greg Wood e-mail: (greg.wood@mmu.ac.uk). 
Highlights

Combined action observation and motor imagery facilitates visuomotor adaptation Updating of the internal forward model can be advanced by an $\mathrm{AO}+\mathrm{MI}$ intervention Internal modelling deficits in children with DCD are reflected in their eye-movements $\mathrm{AO}+\mathrm{MI}$ intervention improved eye-hand coordination and movement kinematics $\mathrm{AO}+\mathrm{MI}$ has potential as an intervention technique for use with children with $\mathrm{DCD}$

3

4


The internal modelling deficit (IMD) hypothesis suggests that motor control issues associated with Developmental Coordination Disorder (DCD) are the result of impaired predictive motor control. In this study, we examined the benefits of a combined action observation and motor imagery $(A O+M I)$ intervention designed to alleviate deficits in internal modelling and improve eye-hand coordination during a visuomotor rotation task. Twenty children with DCD were randomly assigned to either an AO+MI group (who watched a video of a performer completing the task whilst simultaneously imagining the kinaesthetic sensations associated with action execution) or a control group (who watched unrelated videos involving no motor content). Each group then attempted to learn a $90^{\circ}$ visuomotor rotation while measurements of completion time, eye-movement behaviour and movement kinematics were recorded. As predicted, after training, the $\mathrm{AO}+\mathrm{MI}$ group exhibited quicker completion times, more target-focused eye-movement behaviour and smoother movement kinematics compared to the control group. No significant after-effects were present. These results offer further support for the IMD hypothesis and suggest that $A O+M I$ interventions may help to alleviate such deficits and improve motor performance in children with DCD.

Keywords: Internal model deficits, motor learning, mental simulation, eye-movements, eyehand coordination, visuomotor rotation 


\section{Introduction}

Developmental coordination disorder (DCD) is a neurodevelopmental disorder that is estimated to affect between $1.7 \%$ and $6 \%$ of children worldwide (American Psychiatric Association [APA], 2013). The condition is categorised as a marked impairment in the development of motor coordination that interferes with activities of daily living. These impairments are below the level expected for the child's chronological age and must not be attributable to other neurological conditions, sensory problems, or low intelligence (APA, 2013). While the aetiology of DCD is not fully understood, one suggestion is that these motor control issues are the result of impaired predictive motor control, stemming from disrupted cognitive representations of movement. This has been labelled as the internal modelling deficit (IMD) hypothesis (Wilson \& Butson, 2007; Wilson, Ruddock, SmitsEngelsman, Polatajko, \& Blank, 2013).

According to Wolpert (1997), internal models are neural representations of the external world that are used to calculate and adjust movements by predicting their expected sensory consequences. These predictions are made by comparing the body's current state to an efference copy of the motor command, which contains predicted movement trajectories and associated bodily sensations (Kawato, 1999). As typical sensorimotor learning develops, the incongruence between predicted and actual movement sensations are diminished or are used to guide skilful online adjustments, increasing movement coordination. Conversely, difficulty in the generation or implementation of predictive models of action leads to slow, effortful, inaccurate, and uncoordinated movements that are overly dependent on visual feedback (Deconinck et al., 2006; Wilson et al., 2013). These difficulties are characteristic of children with DCD (for a review, see Adams, Lust, Wilson, \& Steenbergen, 2014) and are commonly observed in visuomotor adaptation tasks and through deficits in motor imagery ability.

Visuomotor adaptation is a form of sensorimotor learning that consists of participants learning to adapt, or correct for, an external (often visual) perturbation. One example of this is through visuomotor rotation tasks where the motion of a cursor is rotated by a given angle with respect to the motion of the mouse controlling it. The rate of adaptation to this rotation is a measurement of the direct-effects of the development of an internal model between motor movements and the spatial goal of the task (Wang \& Lei, 
2015). The examination of after-effects (where the rotation is taken away) is a measure of how established the internal model actually is (Krakauer, 2009), with greater after-effects suggesting a more well-established internal model. After-effects are the unintentional remains of compensatory strategies used to adapt to a novel visuomotor workspace that are present when the performer is reintroduced to an environment in which the use of such strategies is not necessary (Ong \& Hodges, 2010).

Using a line drawing task on a digitised tablet, Kagerer, Bo, Contreras-Vidal and Clark (2004) asked children with and without DCD to perform a $45^{\circ}$ visuomotor rotation task and examined both direct-effects and after-effects. Results revealed that children with DCD were less affected by the visuomotor rotation and showed no after-effects. This suggested that they had a less well-defined internal model compared to the typically developing children. In a follow-up study, using a more complex $60^{\circ}$ visuomotor rotation, Kagerer, Contreras-Vidal, Bo and Clark (2006) showed that children with DCD updated their internal model more effectively during exposure to an abrupt $60^{\circ}$ visuomotor rotation compared to a more gradual rotation (i.e., increasing rotations of $10^{\circ}$ every 21 trials until a rotation of $60^{\circ}$ was achieved). These results suggest that the adaptation process in children with DCD is mediated by the complexity of the visuomotor perturbation, due to an impaired capacity to use small error signals to modify an internal model. Similar findings have also been reported in prism adaptation experiments, in which visual feedback is displaced using prism glasses that deflect vision laterally during throwing tasks (Brookes, Nicolson, \& Fawcett, 2007; Cantin, Polatajko, Thach, \& Jaglal, 2007).

Internal modelling deficits have also been evidenced in research examining the motor imagery ability characteristics of children with DCD. Motor imagery is the process of mentally rehearsing actions, typically without overt action or physical output (Jeannerod, 2001). Motor imagery is thought to access the same neural representation of a movement as that used in predictive modelling. This link to internal models is evidenced through research showing that motor imagery activates similar brain regions to those involved in motor skill planning and execution (Hardwick, Caspers, Eickhoff, \& Swinnen, 2018), evokes similar eye-movement patterns (Causer, McCormick, \& Holmes, 2013) and similar temporal congruence (i.e., mental chronometry) between imagined and executed actions (Guillot, Hoyek, Louis, \& Collet, 2012). In accordance with the IMD hypothesis, individuals with DCD 
exhibit impairments in mental chronometry ability (Ferguson, Wilson \& Smits-Engelsman, 2015), reduced ability to imagine egocentric transformations of the body (Barhoun et al., 2019), an impairment in the accuracy of motor imagery (Fuchs \& Caçola, 2018) and reduced corticospinal excitability during motor imagery (Hyde et al., 2018).

Mental simulation techniques like motor imagery and action observation (i.e., the structured observation of action execution) have been proposed to be effective interventions that target internal model deficits (Adams, Lust \& Steenbergen, 2018). These interventions have shown promise in improving movement outcomes in sporting tasks (Cumming \& Ramsey, 2009) and for clinical conditions like Parkinson's disease (Caligiore, Mustile, Spalletta, \& Baldassarre, 2017), stroke (Ertelt \& Binkofski, 2012; ZimmermannSchlatter, Schuster, Puhan, Siekierka, \& Steurer, 2009) and for children with cerebral palsy (Buccino et al. 2018). It has also been suggested that mental simulation techniques may be beneficial for children with DCD (Adams et al., 2018) and a small number of studies have reported positive outcomes. For example, Wilson, Thomas and Maruff (2002) found that motor imagery training was equally as effective as traditional perceptual motor training for developing motor skills, particularly with children with severe DCD (Wilson, Adams, Caeyenberghs, Thomas, Smits-Engelsman \& Steenbergen, 2016). Finally, Adams, SmitsEngelsman, Lust, Wilson and Steenbergen (2017) reported clinically meaningful changes in motor skill proficiency after an intervention that included separate aspects of action observation preceding motor imagery for children with DCD.

Recent research has proposed that combining action observation with concurrent motor imagery of the same action (AO+MI: Eaves, Riach, Holmes, \& Wright, 2016; Vogt, Di Rienzo, Collet, Collins, \& Guillot, 2013) may lead to improved behavioural outcomes compared to either simulation technique performed in isolation (Bek, Gowen, Vogt, Crawford \& Poliakoff, 2019; Romano Smith, Wood, Coyles, Roberts \& Wakefield, 2019; Romano-Smith, Wood, Wright \& Wakefield, 2018; Scott, Emerson, Dixon, Tayler \& Eaves, 2019). The rationale for combining these techniques stems from neurophysiological studies which have identified that $\mathrm{AO}+\mathrm{MI}$ produces increased activity in cortical areas linked to movement planning and execution, compared to either $\mathrm{AO}$ or $\mathrm{MI}$ performed separately (e.g., Wright, Williams \& Holmes, 2014, for a review see Eaves et al., 2016). Recent evidence has suggested that such activity may be related to specific ways in which action observation 
and motor imagery help to develop internal models (Kim, Frank, \& Schack, 2017). Specifically, action observation has been shown to promote the reorganization of frontoparietal cortex as visual information is mapped onto motor circuits (Apšvalka, Cross, \& Ramsey, 2018) and may help to develop the sequencing and timing of basic action concepts (Wright, Wood, Eaves, Bruton, Frank \& Franklin, 2018). These basic action concepts are smaller components of mental representations that are related functionally and biomechanically to the successful execution of a motor skill (Frank, Land \& Schack, 2013) and are encoded in long-term memory to guide motor skill execution (Schack \& Mechsner, 2006). Kinaesthetic imagery has been shown to expedite the development of the internal model by improving the prediction of sensory consequences of the imagined movements (Kilteni, Andersson, Houborg \& Ehrsson, 2018). Based on this evidence, and that which suggests children with DCD struggle with visual imagery, it is possible that combining both techniques through $\mathrm{AO}+\mathrm{MI}$ will provide a more effective intervention that promotes the development of internal models and facilitates motor skill acquisition.

In a recent study that brought these areas together, Marshall, Wright, Holmes and Wood (2019) examined the efficacy of an AO+MI intervention in facilitating adaptation to a visuomotor rotation task in healthy adults. Specifically, participants wore eye-tracking equipment whist performing an $180^{\circ}$ visuomotor rotation task (i.e., leftward movements of the hand resulted in rightward movements of the cursor and vice-versa) at pre-test, during 20 intervention trials, and post-test. Results indicated that, relative to a control group, participants who engaged in $\mathrm{AO}+\mathrm{Ml}$ improved visuomotor adaptation (i.e., reduced task completion time) and alleviated the early reliance on visual feedback to control the cursor movement. This early reliance on visual feedback control is linked to the need to establish effective sensorimotor mapping rules (i.e., an internal model) related to motor commands, sensory outcomes and cursor movement (Sailer, Flanagan \& Johansson, 2005). As internal models become established, vision is used in a more feedforward manner (i.e., targetfocused) that supports the planning and control of manual action, indicative of task expertise (Land, 2009). Marshall et al.'s (2019) findings indicate that AO+MI interventions can facilitate the development of internal models and that this developmental process can be measured through changes in task-specific eye-movement behaviours. 

movements (e.g., Debrabant, Gheysen, Caeyensberghs, Van Waelvede \& Vigerhoets, 2013), no studies have explored eye-movements during the adaptation to visuomotor rotation in children with DCD. This is important as further support for the IMD hypothesis may be gained from an exploration of eye-movement behaviours of children with DCD during adaptation to visuomotor rotation. Furthermore, no studies have explored the efficacy of $\mathrm{AO}+\mathrm{MI}$ for facilitating this process in this population. As individuals with $\mathrm{DCD}$ exhibit poor motor imagery ability, combining action observation with kinaesthetic imagery may be an effective intervention that provides accurate visual and temporal movement cues while enabling cognitive resources to be devoted to the generation of kinaesthetic imagery associated with the observed movement (Eaves et al., 2016). As visuomotor adaptation has been used with children with DCD previously, it is an ideal paradigm to assess the efficacy of $\mathrm{AO}+\mathrm{MI}$ interventions for improving internal model deficits.

The aim of this experiment was to extend previous research on visuomotor adaptation and mental simulation in children with $D C D$ by examining the utility of an AO+MI intervention for facilitating visuomotor adaptation and eye-hand coordination. Based on previous evidence (Marshall et al., 2019), it was hypothesised that AO+MI training would help to overcome deficits in internal modelling and produce a significant improvement in visuomotor adaptation task performance, underpinned by the facilitation of more predictive (i.e., target-focused) eye-movement behaviours, shorter cursor path lengths, and smoother movement kinematics. Finally, it was predicted that AO+MI training would produce significant after-effects when participants repeated the task with no rotation applied, indicating the more extensive development of the internal model (Kagerer et al. 2006).

\section{Method}

\subsection{Participants}

Twenty children aged 7 to 11 years (13 male, 7 female; age $M=9.0, S D=1.45$ years) with confirmed or suspected DCD were recruited through local DCD support groups. Potential participants were first screened using the revised version of the Developmental Coordination Disorder Questionnaire (DCDQ: Wilson, Kaplan, Crawford \& Roberts, 2007) and those who were identified as potentially having DCD (i.e., scores within the range of 15- 
55) were then invited to a testing session where they also completed the Movement Assessment Battery for Children-2 (MABC-2: Henderson, Sugden \& Barnett, 2007). Only children who scored at or below the $5^{\text {th }}$ percentile on the MABC-2 and who, based on parent reports, did not suffer from any other general medical condition known to affect sensorimotor function (e.g., cerebral palsy, hemiplegia, or muscular dystrophy) and had no diagnosis of learning difficulties or ADHD, were asked to take part in the study. Parents and children provided written informed consent and assent, respectively, prior to taking part. The experimental procedures were granted ethical approval by the institutional ethics committee prior to testing.

\subsection{Task}

Participants performed a virtual radial Fitts task. For this task, a $90^{\circ}$ counterclockwise visual feedback rotation was used that resulted in stylus movements along the $x$ axis producing equivalent cursor movements along the $y$-axis and vice versa. This rotation resulted in upward movement of the stylus producing rightward cursor movement, rightward stylus movement produced a downward cursor movement, a downward stylus movement produced a leftward cursor movement and a leftward stylus movement produced an upward cursor movement. The goal of the task was to use a stylus to guide a cursor from a central home square to a yellow highlighted target square and then back to the home square (see Figure 1). Six targets were presented sequentially from left to right with the next target becoming highlighted each time the cursor returned to the central square. Based on a similar design used by Heremans et al. (2011), all the target positions were visible throughout the task in an arc radiating out at a distance of $170 \mathrm{~mm}$ from the central square. One full trial consisted of all six targets being successfully hit and the cursor returning to the central square each time (totalling 12 target hits). Unity3D (Unity Technologies, San Francisco, CA) software was used to present the experimental task, to collect data in relation to cursor movement $(80 \mathrm{~Hz})$ and to record task completion time.

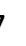
58 
Figure 1. Image showing the experimental set-up (a) and the visuomotor adaptation task shown in the AO+MI video (b). The red circle around the cursor square represents the participant's point of gaze and the yellow squares represent the target squares. The white square in the bottom/centre of the image represents the 'home' square.

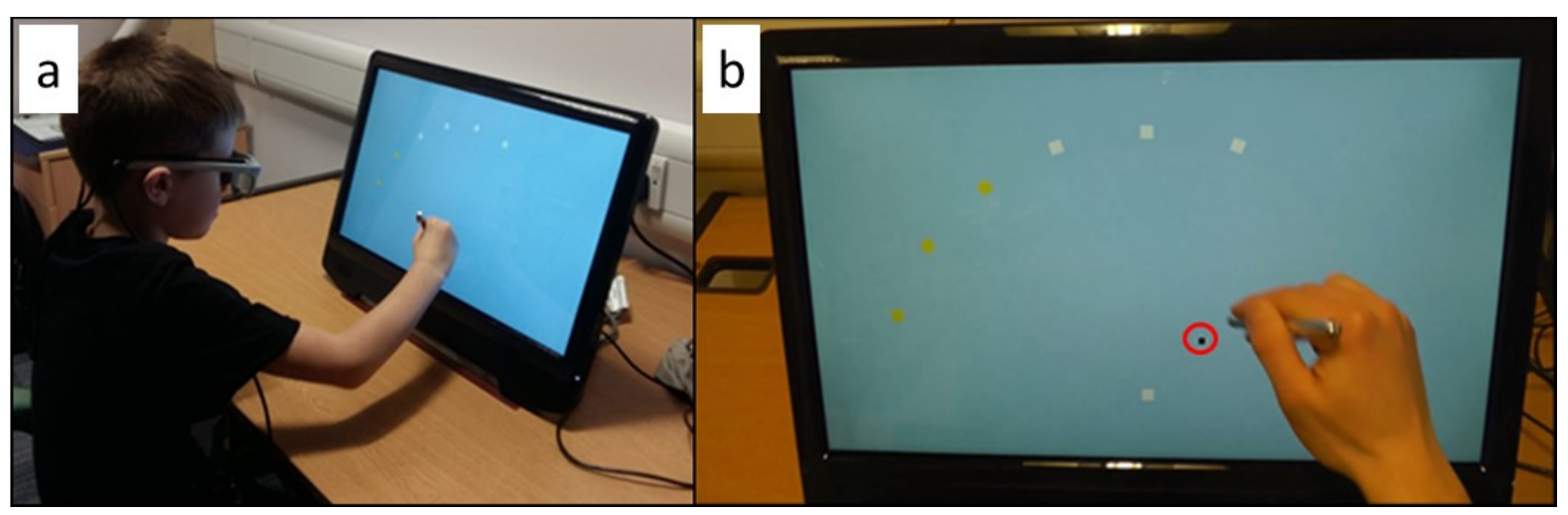

\subsection{Apparatus}

Testing was performed on a vertically-oriented Dell ST2220T touchscreen monitor (Dell, Round Rock, TX) with a $480 \mathrm{~mm} \times 270 \mathrm{~mm}$ visual display, situated $210 \mathrm{~mm}$ from the edge of the table where the participant was seated (Figure 1). Eye-movements were monitored using ETG 2w eye tracking glasses and iView ETG 2.7 software (SMI, Teltow, Germany). The system comprises a pair of lightweight glasses that track participants' binocular eye-movements at a sampling rate of $60 \mathrm{~Hz}$ with a gaze position accuracy of $0.5^{\circ}$. The eye tracking glasses were calibrated for each participant prior to each trial by instructing them to fixate on points on a calibration grid that represented the spatial arrangement of the target sequences. If, during the session, the quality of the calibration was deemed by the experimenter to have deteriorated then the calibration procedure was repeated before testing continued.

\subsection{Procedure}




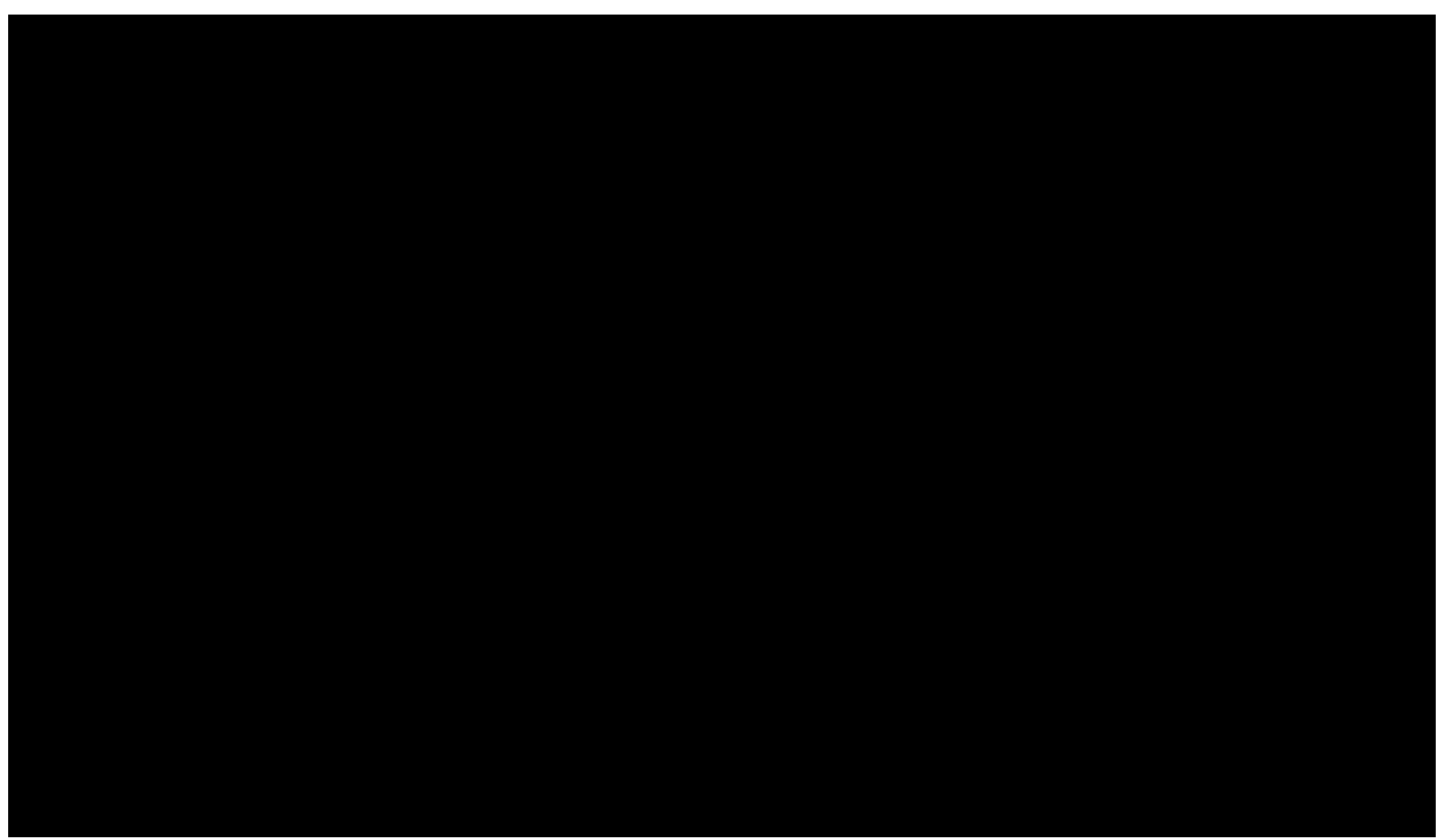

\subsubsection{Pre-test: No rotation}

Participants were first calibrated to the eye-tracker before performing two practice trials (totalling 24 target hits) of the task with no visuomotor rotation applied in order to familiarise themselves with the stylus, goal of the task, and experimental set-up.

Participants then performed three pre-test trials (totalling 36 target hits) of the task with no visuomotor rotation applied which would be compared to any after-effects postintervention. Throughout each phase of the experiment, the number of trials and target hits was based on those used by Kagerer et al. (2006) as this study used a similar visuomotor task to investigate visuomotor adaptation in children with DCD. Participants were instructed to perform the task as quickly and accurately as possible on each trial.

\subsubsection{Pre-test: Rotation}

Once participants had completed their practice trials, they then performed one trial

298 (totalling 12 target hits) of the task with the $90^{\circ}$ visuomotor rotation applied. Prior to starting this trial, participants were informed that, although the task looked the same and still had the same goal, the cursor would move differently. Each participant was given a maximum of three minutes to hit all of the presented targets. If all the targets had not been hit during this time, 180 seconds was recorded as the trial completion time, along with the 
number of targets successfully hit. Of the 20 participants, 14 reached the 180 second limit on the pre-test $(M=164.85, S D=26.61)$. The three-minute maximum allowed for some control over the amount of exposure participants had to the novel visuomotor environment prior to training. Immediately after completing the pre-test rotation trial, participants started the training intervention to which they had been randomly assigned.

\subsubsection{Intervention Groups}

$A O+M I$. Participants in the $A O+M I$ group (six male, four female; age $M=9.0, S D=$ 1.56 years) performed motor imagery of executing the task whilst they simultaneously observed a series of videos of a novice, typically developing, adult performer completing the same visuomotor rotation task. The video series consisted of three videos recorded at different stages of the learning experience as they performed 50 trials of the task. These stages were determined based on the number of trials completed by a child of similar age and were identified as: Early (trials 1 to 10 ), Mid (trials 11 to 30 ), and Late (trials 31 to 50). Each video was selected to represent the natural progression of adaptive behaviour as the child became more accomplished at the task (see Table 1 for a visualisation of the cursor path associated with these stages). The use of a series of videos for the $\mathrm{AO}+\mathrm{MI}$ intervention was included as established models of motor imagery recommend that the motor imagery experience should adapt as learning progresses to reflect a learner's level of physical experience (Holmes \& Collins, 2001). In addition, visuomotor adaptation studies using observational learning have also used videos that show progressive changes in the model's performance (Lei, Bao \& Wang, 2016). Each video was filmed from the same first-person perspective, recorded from the scene camera of the eye-tracker, and showed only the touchscreen monitor and the novice performer's hand moving the stylus over the screen in order to guide the cursor to each target (see Figure 1b). At the start of each video, a motor imagery script was presented in written form on the screen along with an audio-recorded narration. This script was slightly different for each video in order to reflect the adaptations made by the novice performer as their training progressed (see Table 1). Only kinaesthetic imagery instructions were provided because visual information was provided in the video, typical of AO+MI interventions (Eaves et al., 2016).

After each $\mathrm{AO}+\mathrm{MI}$ trial, participants immediately performed a physical practice trial as previous research has suggested that observational learning alone is not enough to 
update an internal model of the visuomotor environment and at least some amount of physical practice is required (Ong \& Hodges, 2010; Ong, Larssen \& Hodges 2012; Lei et al., 2016). This resulted in this intervention consisting of $21 \mathrm{AO}+\mathrm{MI}$ trials (totalling 252 target hits) and 21 physical practice trials (totalling 252 target hits), separated into three blocks of practice (see Figure 2 ). Rest periods ( 2 mins) were given after every block and the eyetracking equipment was checked for calibration before the start of each trial.

Control. Participants in the control group (seven male, three female; age $M=9.0, S D$ $=1.41$ years) watched 42 second clips of a nature documentary that contained no human motor content (Scott et al., 2019) followed by an immediate physical practice trial. The duration of video clips was chosen in order to represent a total viewing time that was equivalent to the total duration of the $\mathrm{AO}+\mathrm{MI}$ videos. These trials were also divided into three blocks of seven video and immediate physical practice trials and in total, participants in this group physically performed 21 trials of the task (totalling 252 target hits). Rest periods ( 2 mins) were given after every block and the eye-tracking equipment was checked for calibration before the start of each trial.

\subsubsection{Post-test: Rotation}

Each participant completed a final rotation trial (totalling 12 target hits) as a posttest that was identical to the pre-test conditions. Each participant was again given a maximum of three minutes to hit all of the presented targets.

\subsubsection{Post-test: No Rotation}

Participants performed three trials of the task (totalling 36 target hits) with no visuomotor rotation, identical to pre-test conditions, to assess the presence of any aftereffects. After this was completed, participants and their parents were debriefed and thanked for their participation. 
Table 1. AO+MI instructions for each training stages of the intervention and the plotted cursor paths of the model to give an illustration of the kinematic information represented in each action observation video.

Instructions
Stage "I am watching the video on the screen.
The hand in the video is mine and I am
making the movements that I see. I can
feel myself holding the pen and I can feel
my arm and hand moving the cursor to
the yellow squares"
"I am watching the video on the screen.
The hand in the video is mine and I am
making the movements that I see. I can
feel myself holding the pen and I can feel
my arm and hand moving the cursor in
circles towards the yellow squares"
"I am watching the video on the screen.
The hand in the video is mine and I am
making the movements that I see. My
movements are steady and accurate. I can
feel myself holding the pen and I can feel
my arm and hand moving the cursor in
oval patterns towards the yellow
squares"

\section{Measures}

\subsection{Completion time}

The time taken (in seconds) to finish the entire trial (12 target hits), from leaving the home square at the start to returning to the home square after hitting the sixth target, was used as a measure of completion time.

\subsection{Target-locking score}

Each pre-test and post-test trial for each participant was analysed using the BeGaze 3.7 software (SMI, Teltow, Germany). In addition, the $1^{\text {st }}, 3^{\text {rd }}, 5^{\text {th }}$, and $7^{\text {th }}$ trials from each training block were also analysed. Targets were defined as the six outboard target squares and the central home square. Fixations were defined as gaze dispersed over less than $3^{\circ}$ of visual angle for a minimum of $80 \mathrm{~ms}$. A target-locking score was then calculated by 
subtracting the percentage of cursor fixation time from the percentage of target fixation time to create a ratio measure of the allocation of visual attention. This method has previously been used to determine the gaze control of participants performing visuomotor adaptation tasks (Marshall et al., 2019), surgical tasks (Wilson, McGrath, Vine, Brewer, Defriend \& Masters , 2010), and tasks involving the control of a prosthetic hand (Parr, Vine, Harrison \& Wood, 2018; Parr, Vine, Wilson, Harrison \& Wood, 2019). Using this method, a more positive score reflects more time fixating on targets whereas a negative score reflects more time spent fixating the cursor. A score of ' 0 ' reflects equal time spent fixating the cursor and targets and represents a 'switching strategy'.

\subsection{Movement Kinematics}

For each trial, cursor movements were filtered using a 2nd order dual lowpass Butterworth filter with an $8 \mathrm{~Hz}$ cut off frequency. The filtered data was then processed with custom written Matlab 2017b (MathWorks Inc, Natick, MA) routines.

\subsection{Total Path length}

As children with DCD are thought to persist with ineffective movement strategies (Biotteau, Chaix \& Albaret, 2016), we measured total path length $(\mathrm{mm})$ to gain a quantifiable representation of the movement strategies that children were using in both groups. Total path length was calculated between sampled pairs of $x$ and $y$ coordinates using the following formula where $x_{1}, x_{2}$ and $y_{1}, y_{2}$ represent points along the $x$ and $y$ axes respectively. The total units of distance $(\mathrm{mm})$ for each sampled point were then summed to provide a total path length for each trial.

$$
\text { Path length }=\sqrt{\left(x_{2}-x_{1}\right)^{2}+\left(y_{2}-y_{1}\right)^{2}}
$$

\subsection{Normalised Jerk}

For each trial, jerk was calculated as a measure of movement smoothness. As jerk varies according to both the duration and size of a movement, these data was normalised using the following formula where $j$ refers to jerk and $t$ to time:

$$
\text { Normalised jerk }=\sqrt{\left(1 / 2 \int d t j^{2}(t) \times \frac{\text { duration }^{5}}{\text { length }^{2}}\right)}
$$


403

404

405

406

407

408

409

410

411

412

413

414

415

416

417

418

419

420

421

422

423

424

425

426

427

428

429

430

431

This calculation produces a unit-free measure that can be used to compare movements of different sizes and durations (Teulings et al., 1997; Kagerer et al., 2006).

\subsection{After-effects}

The presence of after-effects following the adaptation training was assessed by calculating completion time, target-locking score, total path length, and normalised jerk on the no rotation trials pre and post intervention. In addition, the length and root mean square error (RMSE) of the path to the first target was also calculated in order to identify any initial after-effects before they were washed out over subsequent target hits. RMSE is a measure of the spatial deviation from a direct vector between home and target (Kagerer et al., 2004; 2006).

\subsection{Data analysis}

Due to the data for completion time and mean total path length violating the assumption of normality, these variables were successfully log transformed. Separate 2 (Group: AO+MI, control) x 5 (Time: Pre-test, T1, T2, T3, Post-test) mixed measures ANOVAs were performed on participant's completion time, gaze control, mean path length, and normalised jerk. Significant interactions were followed up with Bonferroni corrected pairwise comparison that compared each group at each time point (Pre-test, T1, T2, T3, Post-test). To assess the presence of after-effects, a 2 (Group: AO+MI, control) x 2 (Pre-test vs. Post-test) mixed measures ANOVA was conducted for pre and post no rotation trials (Kagerer et al., 2006). For all analyses, where sphericity was violated, Greenhouse-Geisser corrections were applied. Effect sizes are reported as partial eta squared $\left(\eta p^{2}\right)$, and the alpha level for statistical significance was set at 0.05 .

\section{Results}

\subsection{Completion time}

The ANOVA revealed significant main effects for time, $F(2.41,43.35)=152.45, p<$ $.001, \eta_{\mathrm{p}}{ }^{2}=.89$, and group, $F(1,18)=11.53, p=.003 \eta_{\mathrm{p}}{ }^{2}=.39$, which were superseded by a significant interaction effect, $F(2.41,43.55)=3.97, p=.020, \eta_{p}{ }^{2}=.18$. As expected, post-hoc comparisons revealed no significant difference between groups at pre-test $(p=.699)$ or T1 ( $p$ $=.172$ ), but the $\mathrm{AO}+\mathrm{MI}$ group produced significantly faster completion times than the 
control group at $\mathrm{T} 2(p=.002), \mathrm{T} 3(p=.007)$ and post-test $(p=.009)$. These data are presented in Figure 3a.

\subsection{Target-locking score}

The ANOVA revealed significant main effects for time, $F(2.01,36.22)=114.78, p<$ $.001, \eta_{p}{ }^{2}=.86$, and group, $F(1,18)=22.89, p<.001, \eta_{p}{ }^{2}=0.56$, which were superseded by a significant interaction, $F(2.01,36.22)=4.26, p=.022, \eta_{p}{ }^{2}=.19$, for target-locking score. As expected, post-hoc comparisons revealed no significant difference between groups at pretest $(p=.33)$, but the AO+MI group had a significantly greater TLS at T1 $(p<.001), \mathrm{T} 2(p<$ $.001)$, Т3 $(p=.002)$ and post-test $(p=.012)$. These data are presented in Figure $3 \mathrm{~b}$.

\subsection{Movement kinematics}

All pre-test kinematic data for one participant in the $\mathrm{AO}+\mathrm{MI}$ group was removed prior to analysis due to technical issues with the touch screen that meant the cursor functioned correctly but the values generated were erroneous.

\subsection{Total path length}

The ANOVA revealed a significant main effect for time, $F(1.94,33.01)=12.53, p<$ $.001, \eta_{\mathrm{p}}^{2}=.42$, indicating that both groups produced shorter cursor paths as training progressed. There was no significant main effect for group, $F(1,17)=3.91, p=.064, \eta_{\mathrm{p}}{ }^{2}=$ .18 , and, unexpectedly, no significant interaction was found, $F(1.94,33.01)=2.65, p=.087$, $\eta_{\mathrm{p}}{ }^{2}=.14$. These data are presented in Figure $3 c$. A visual representation of path length illustrating the strategies that participants typically used is presented in Figure 4.

\subsection{Normalised Jerk}

The ANOVA revealed a significant main effect for time, $F(4,68)=11.79, p<.001, \eta_{p}{ }^{2}$ $=.41$, indicating both groups exhibited an increase in movement smoothness throughout the training. A significant main effect for group was also revealed, $F(1,17)=31.98, p<.001$, $\eta_{\mathrm{p}}{ }^{2}=.65$, indicating that the movements of the control group were significantly more jerky $(M=8.98, S D=4.02)$ compared to the movements of the AO+MI group $(M=5.28, S D=$ 1.77). In contrast to our predictions, no significant interaction was found, $F(4,68)=.79, p=$ $.536, \eta_{p}^{2}=.05$. These data are presented in Figure $3 \mathrm{~d}$. 
The ANOVA revealed no significant main effects or interactions between groups for

462 all after-effect variables measured (see Table 2). 
Figure 3. Mean completion time (a), mean target-locking score (b), total cursor path length (c) and normalised jerk (d) for both groups across pre-test, training blocks (T1, T2, T3) and post-test.

465
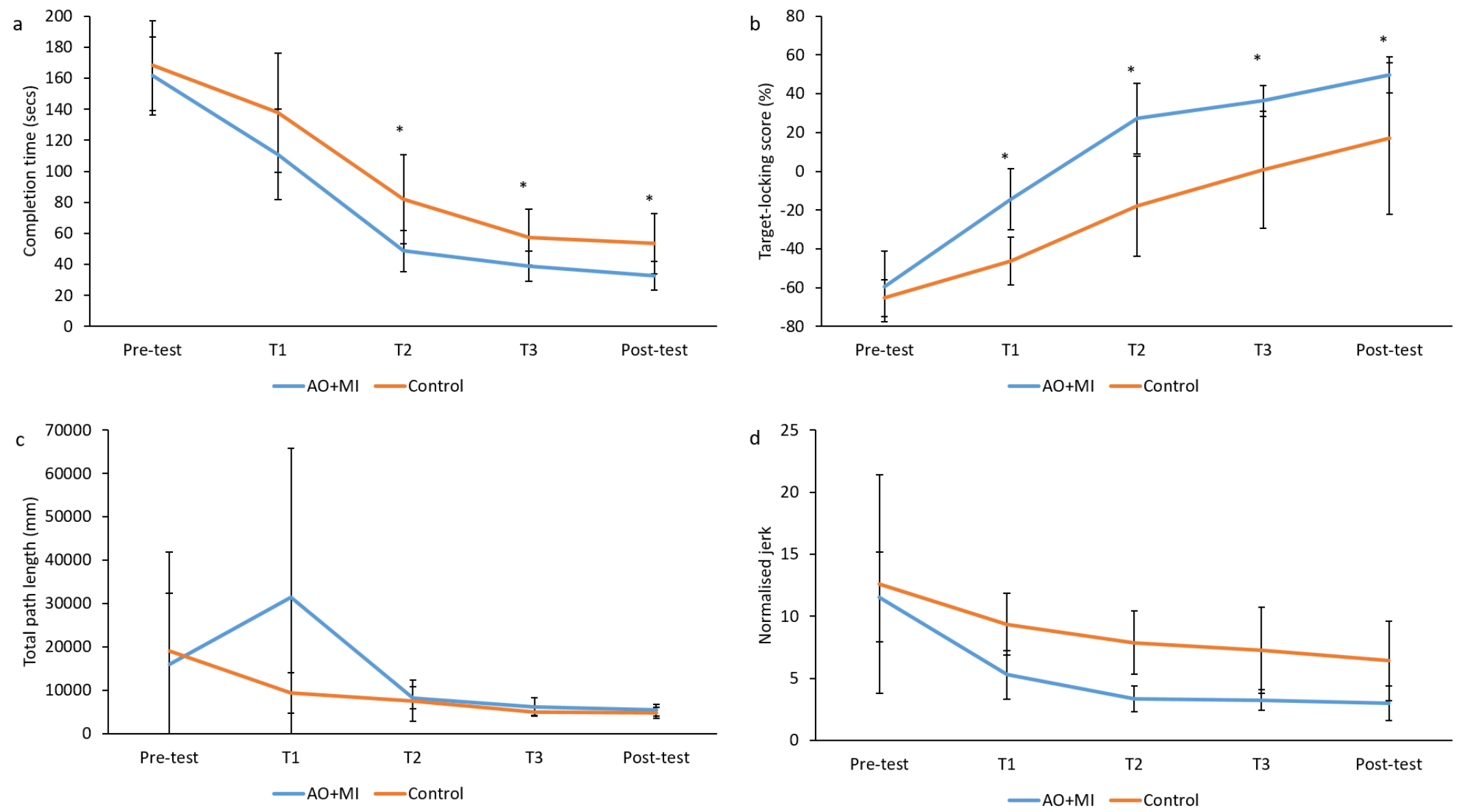
477

Table 2. After-effects data showing the completion time, target-locking score and kinematic data (SD) for each intervention group at pre-test and post-test with no rotation present.

\begin{tabular}{|c|c|c|c|c|c|c|c|c|}
\hline & \multicolumn{2}{|c|}{$\mathrm{AO}+\mathrm{MI}$} & \multicolumn{2}{|c|}{ Control } & \multicolumn{4}{|c|}{ Inferential Statistics } \\
\hline & Pre & Post & Pre & Post & & df & $F$ & $p$ \\
\hline Completion time & 14.66 & 17.04 & 14.49 & 17.27 & Time & 1,18 & 2.42 & .138 \\
\hline (seconds) & (5.90) & (5.31) & $(4.82)$ & $(6.78)$ & Group & 1,18 & .00 & .989 \\
\hline & & & & & Interaction & 1,18 & .02 & .904 \\
\hline
\end{tabular}

$\begin{array}{cccccrrrr}\text { Target-locking } & 64.01 & 61.61 & 62.34 & 55.40 & \text { Time } & 1,18 & .47 & .504 \\ \text { score (\%) } & (23.01) & (31.50) & (17.98) & (22.47) & \text { Group } & 1,18 & .21 & .655\end{array}$

\begin{tabular}{|c|c|c|c|c|c|c|c|c|}
\hline \multirow{3}{*}{$\begin{array}{l}\text { Total path length } \\
\text { (mm) }\end{array}$} & 2749.55 & 3262.23 & 2866.35 & 2884.36 & Time & 1,18 & 2.14 & .161 \\
\hline & (171.05) & (1035.77) & $(246.44)$ & (672.84) & Group & 1,18 & .36 & .558 \\
\hline & & & & & Interaction & 1,18 & 1.86 & .190 \\
\hline \multirow[t]{3}{*}{ Normalised jerk } & 3.21 & 2.02 & 2.99 & 2.68 & Time & 1,18 & 7.44 & .014 \\
\hline & $(0.46)$ & $(0.53)$ & $(0.94)$ & (1.11) & Group & 1,18 & .78 & .389 \\
\hline & & & & & Interaction & 1,18 & 2.60 & .125 \\
\hline \multirow{3}{*}{$\begin{array}{l}\text { First path length } \\
\qquad(\mathrm{mm})\end{array}$} & 265.76 & 338.28 & 254.82 & 270.23 & Time & 1,18 & 3.39 & .082 \\
\hline & $(65.16)$ & (122.90) & (49.99) & (56.05) & Group & 1,18 & 2.30 & .147 \\
\hline & & & & & Interaction & 1,18 & 1.43 & .248 \\
\hline \multirow{3}{*}{$\begin{array}{l}\text { First path RMSE } \\
\text { (mm) }\end{array}$} & 9.81 & 12.09 & 10.45 & 10.94 & Time & 1,18 & 2.90 & .106 \\
\hline & $(0.55)$ & (4.05) & $(2.84)$ & (2.99) & Group & 1,18 & .06 & .802 \\
\hline & & & & & Interaction & 1,18 & 1.21 & .286 \\
\hline
\end{tabular}


Figure 4. A visual representation of cursor paths and respective completion times (seconds) produced by two participants during the AO+MI (top) and control (bottom) training interventions. These participants were chosen as their post-test completion times were similar to the overall group mean completion times for each $A O+M I(M=53.76, S D=22.34)$ and Control group $(M=26.15, S D=14.82)$ at post-test.

\section{AO+MI}

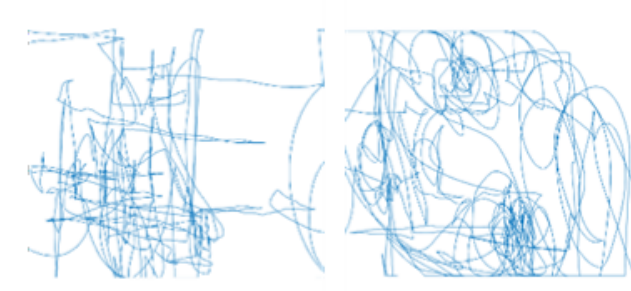

Pre-test

180 secs

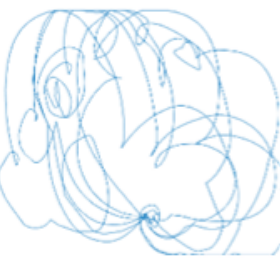

T2

47.17 secs

Control

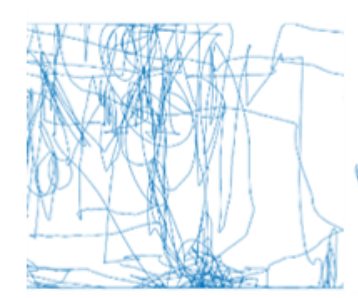

Pre-test

180 secs
T1

76.00 secs

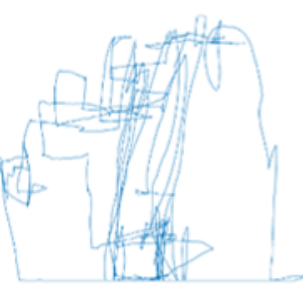

T1

120.61 secs

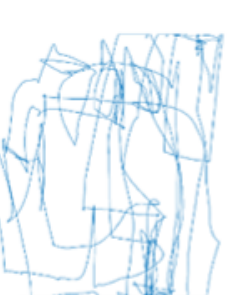

T2

72.56 secs

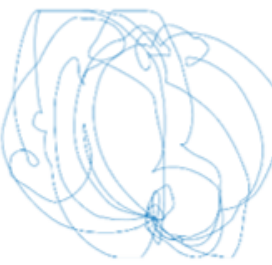

T3

40.62 secs
Post-test

28.72 secs

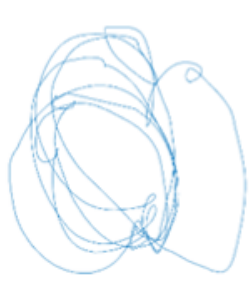

28.72 secs 


\section{Discussion}

The aim of this experiment was to extend previous research on visuomotor adaptation and mental simulation in children with DCD by examining the benefits of an $\mathrm{AO}+\mathrm{MI}$ intervention for facilitating visuomotor adaptation and eye-hand coordination. Based on the assumption that the impairments associated with DCD are the result of deficits in internal modelling, it was predicted that a dual-simulation technique incorporating the simultaneous performance of both $\mathrm{AO}$ and $\mathrm{MI}$ would facilitate the development of internal models, improve visuomotor adaptation, and optimise both eye-movement behaviour and movement kinematics. The results of this experiment provide some support for these hypotheses. First, as predicted, the $\mathrm{AO}+\mathrm{MI}$ training group produced a significant improvement in task performance (i.e., quicker completion times) compared to the control group. In fact, the AO+MI group performed significantly quicker than the control group by the second training block (T2) and maintained this advantage in the subsequent training block (T3) and post-test phase (Figure 3a). These results are the first to demonstrate that $\mathrm{AO}+\mathrm{MI}$ interventions can aid visuomotor adaptation and support previous research that has shown beneficial effects of $\mathrm{AO}+\mathrm{MI}$ on performance outcomes generally (Bek et al., 2019; Romano-Smith et al., 2018; 2019) and within the DCD population specifically (Scott et al., 2019).

Further evidence that $\mathrm{AO}+\mathrm{MI}$ helped to develop internal models is reflected in the eye-movement data. As eye-movement patterns are shaped by internal models (Hayhoe \& Ballard, 2005), it was expected that any changes in the internal model would be reflected in changes in eye-movement behaviour. As predicted, the eye-movements of the AO+MI group progressed from being predominately used as a feedback resource (i.e., watching the cursor movement) to becoming a feedforward resource (i.e., target-focused) as children became more skilled at the task. Whereas both groups exhibited a predominantly 'cursor-focused' visual strategy at pre-test (target-locking score of approximately -60\%), the $\mathrm{AO}+\mathrm{MI}$ group became almost totally 'target-focused' at post-test (target-locking score of approximately $40 \%)$. In contrast, the control group were unable to progress much beyond a switching strategy between the cursor and target by post-test (target-locking score just above $0 \%$; Figure $3 \mathrm{~b})$. Interestingly, the $\mathrm{AO}+\mathrm{MI}$ group surpassed the development of the control group after the first training block. 
These changes in eye-movement behaviours are consistent with previous research in visuomotor learning (e.g., Sailer et al., 2005) and with recent research showing similar benefits of $\mathrm{AO}+\mathrm{MI}$ training on visuomotor rotation in typically developing adults (Marshall et al., 2019). This early reliance on slower (visual) feedback control is linked to the need to establish effective sensorimotor mapping rules (i.e., an internal model) relating to motor commands, sensory outcomes and cursor movement (Sailer et al., 2005). As skill progresses and sensorimotor mapping rules are developed, cursor movement is controlled by proprioceptive modes of control and vision is freed-up to focus on targets ahead of time (Marshall et al., 2019). Task-specific (goal-directed) eye-movements of this nature support the planning and control of manual action and are indicative of top-down attentional control and task expertise (Land, 2009). Interestingly, children with DCD have shown an inability to develop optimal, task-specific, eye-movement strategies unless explicitly trained to do so (Miles, Wood, Vine, Vickers \& Wilson, 2016; Wood et al., 2017; Slowinski et al., 2019), as evident in our control group. This reliance on vision to monitor movements aligns with evidence from neurological studies that suggests that children with DCD display increased cortical activity in areas related to visuospatial processing and conscious movement control compared to typically developing peers (Zwicker, Missiuna, Harris, \& Boyd, 2010). This shows that deficits in internal modelling are reflected in eye-movement behaviours of children with DCD and that the exploration of eye-movements during motor skill learning may provide an insight into internal model development in this population.

The findings from the kinematic data were less clear. Significant interaction effects in the kinematic variables, corresponding to those seen in the performance and eye movement data, were predicted. No significant interactions were present. In fact, no differences were found in the total path length between groups, indicating that participants used similar path lengths to hit the targets. However, on inspection of the examples of movement strategies used between groups (Figure 4), a number of qualitative differences are evident. First, both groups initially used a strategy almost exclusively based on vertical and horizontal cursor movements. These movements are typical of an early 'exploratory' stage of learning in visuomotor adaptation tasks (Sailer et al., 2005) and are thought to represent individuals freezing degrees of freedom in order to simplify the movement problem. The $\mathrm{AO}+\mathrm{MI}$ intervention facilitated participants to change this strategy to a more 
optimal one (which more than halved their task completion time at T1), whereas the children in the control group seemed to persist with this inefficient strategy almost until the post-test phase. This persistence with an ineffective strategy is typical of children with DCD (Biotteau et al., 2016).

In terms of movement smoothness, the $\mathrm{AO}+\mathrm{MI}$ group were predicted to exhibit significant reductions in jerk after the intervention. This would indicate a better developed internal model, more effective movement planning and, consequently, more smoothly controlled actions. Although the differences elicited by the $A O+M I$ intervention failed to produce a significant interaction, it is clear that the intervention had different, albeit not significant, effects on each intervention group (Figure 3d). This was somewhat reflected in the significant main effect for group that suggested that the $A O+M I$ group participants had significantly less jerk compared to control group participants. Although no group differences were present at pre-test, it is clear that the $\mathrm{AO}+\mathrm{Ml}$ group experienced an increase in the smoothness of their movement (i.e., decreased jerk) throughout the training and post-test compared to the control group. Based on this, and our findings from the performance and eye movement data, it is possible that the $\mathrm{AO}+\mathrm{MI}$ intervention facilitated more effective movement planning and smoother cursor movement owing to a more substantially developed internal model.

The absence of the expected after-effects may undermine our conclusion that $\mathrm{AO}+\mathrm{MI}$ facilitated the development of an internal model. In fact, both groups exhibited less jerk when the rotation was taken away - probably reflecting an overall learning effect or acclimatisation to the equipment. The lack of the expected after-effects is, however, consistent with the results of other studies that have also found no after-effects despite successful visuomotor adaptation (e.g., Ong \& Hodges, 2010; Lei et al., 2016). In studies of children with DCD, both Kagerer et al. (2004) and King et al. (2011) also reported no significant after-effects when using a similar visuomotor rotation task. In fact, to date only one study has shown some evidence of significant after-effects in children with DCD during visuomotor rotation adaptation (Kagerer et al., 2006). While the presence of after-effects is considered evidence for the formation of an internal model, it is uncertain whether the absence of after-effects necessarily means that no internal model was actually developed. For example, previous visuomotor adaptation studies have suggested that the internal 
model can be updated even in the absence of after-effects (Wang \& Lei, 2015). Based on our after-effects data, the extent to which $\mathrm{AO}+\mathrm{MI}$ facilitated the development of the internal model is unclear. However, when considering the direct-effects data (i.e., performance, eyemovements, and kinematics) it is reasonable to suggest that the direct-effects observed in the current experiment provide preliminary support for the formation and ongoing updating of an internal model in children with DCD.

Some limitations of this experiment need to be considered prior to endorsing $\mathrm{AO}+\mathrm{MI}$ as an effective intervention. First, the sample used in the experiment was relatively small. Previous motor imagery studies conducted by Wilson et al. (2002; 2016) employed group sizes of 18 and 12 participants respectively. However, it is important to note that Wilson et al. (2002) included participants who scored at or below the 50th percentile on the MABC test with only 11 children below the $15^{\text {th }}$ percentile, whilst their replication study used the criteria of the $10^{\text {th }}$ percentile (Wilson et al., 2016). In the present study, only children who scored at or below the $5^{\text {th }}$ percentile on the MABC- 2 test were included in data analysis. The more stringent inclusion criterion in this study was selected in order to provide a more representative sample of the DCD population as it is these individuals who benefit most from mental simulation interventions (Wilson et al., 2016). However, due to heterogeneous nature of DCD and the high movement variability associated with the condition, it is possible that this small sample size had a negative influence on the quality of the kinematic data. It is therefore clear that further studies are needed with larger samples sizes before the efficacy of $\mathrm{AO}+\mathrm{MI}$ interventions for the DCD population can be established. Second, the task used was a 2D computer-based task and it is evident that the beneficial performance effects seen here may not transfer to more complex tasks like those required for activities of daily living. Finally, this study did not have a delayed retention test and, therefore, a more thorough examination of the long-term effects of this intervention is required in order to examine AO+MI-induced motor skill consolidation over a longer period.

Despite these limitations, this research offers several theoretical and practical implications that could facilitate future research. Theoretically, these findings offer some support for the IMD hypothesis and extend existing literature by showing, for the first time, that $\mathrm{AO}+\mathrm{MI}$ can be used to alleviate deficits in the development of internal models in children with $D C D$. These results show that the $A O+M I$ group successfully integrated visual- 
spatial information from the $\mathrm{AO}+\mathrm{MI}$ training into their own physical practice and this process facilitated the rate of their adaptation. The action observation component may have allowed participants to map visual information onto motor circuits in order to enhance motor performance (Apšvalka et al., 2018) and helped to develop basic action concepts related to the timing and sequencing of cursor movement (Wright et al., 2018). The kinaesthetic imagery component has been shown to update the proprioceptive components of the internal model that subsequently improve movement planning and control (Kilteni et al., 2018). The development of more elaborate proprioceptive control is indicative of more expert-like motor control that allows vision to be allocated as a feed-forward resource to guide action ahead of time (Sailer et al., 2005), thereby improving performance. Taken together, it is plausible that combining two mental simulation techniques during $A O+M I$ provided a beneficial effect for the formulation and development of internal models of movement control. Without such training, the control group adapted to the visuomotor rotation significantly more slowly, had a less target focused eye-movement strategy, and less effective movement kinematics.

Additionally, DCD is often characterised as a motor learning disorder despite much evidence to the contrary (see Biotteau et al., 2016 for a review). Whilst motor learning for children with DCD is slower than for typically developing children, the present study again demonstrates that while children with DCD may struggle with formulating effective movement strategies themselves, they are well equipped to incorporate or mimic (e.g., Scott et al., 2019; Slowinski et al., 2019) strategies once they are exposed to them. Although our data suggest that AO+MI may be a suitable intervention for this purpose, further examination of the potential neural mechanisms underpinning these effects is needed in future research (Zwicker et al., 2010), and an examination of the additive effects of each action observation and motor imagery component would be important for the design.

From a practical perspective, $\mathrm{AO}+\mathrm{MI}$ interventions appear to offer a suitable adjunct to the physical practice of motor skills for children with DCD. Consequently, AO+MI may be a suitable technique for parent-led interventions that can be performed at home using digital technologies. Previous research with clinical populations has evidenced the benefits of such an approach for learning activities of daily living (Bek et al., 2018), and parental 
involvement has been highlighted as a key factor in ensuring the success of the interventions for DCD (Morgan \& Long, 2012). Future research should therefore explore the feasibility of this approach for children with DCD. Finally, while it is difficult to isolate the contribution of the individual action observation or motor imagery components, combining these techniques into a single intervention may be of particular practical benefit to children with this condition. As individuals with DCD exhibit poor motor visual imagery ability, combining action observation with kinaesthetic imagery may be an effective intervention that provides accurate visual and temporal movement cues while enabling the limited cognitive resources synonymous with the condition (Alloway, 2011) to be devoted to the generation of kinaesthetic imagery associated with the observed movement (Eaves et al., 2016).

In conclusion, these results support the IMD hypothesis as a possible explanation for the coordination impairments associated with $D C D$ and suggest that $A O+M I$ interventions may help children with $D C D$ to overcome such difficulties. Future research with individuals with DCD should examine the efficacy of $A O+M I$ interventions for more complex movements (e.g., sports skills), and for improving functional movements required for activities of daily living.

\section{Declaration of Competing Interest}

The authors declare no conflict of interest

\section{Acknowledgement}

We would like to thank the children and their parents for taking part in this study. 


\section{References}

Adams, I. L., Lust, J. M., \& Steenbergen, B. (2018). Development of motor imagery ability in children with developmental coordination disorder - A goal-directed pointing task. British Journal of Psychology, 109(2), 187-203.

Adams, I. L., Lust, J. M., Wilson, P. H., \& Steenbergen, B. (2014). Compromised motor control in children with DCD: a deficit in the internal model?-A systematic review. Neuroscience \& Biobehavioral Reviews, 47, 225-244.

Adams, I. L., Smits-Engelsman, B., Lust, J. M., Wilson, P. H., \& Steenbergen, B. (2017). Feasibility of motor imagery training for children with developmental coordination disorder-A pilot study. Frontiers in Psychology, 8, 1271.

Alloway, T. P. (2011). A comparison of working memory profiles in children with ADHD and DCD. Child Neuropsychology, 17(5), 483-494.

American Psychiatric Association (2013). Diagnostic and statistical manual of mental disorders ( $5^{\text {th }}$ ed.) Washington DC: Author.

Apšvalka, D., Cross, E. S., \& Ramsey, R. (2018). Observing action sequences elicits sequencespecific neural representations in frontoparietal brain regions. Journal of Neuroscience, 38(47), 10114-10128.

Barhoun, P., Fuelscher, I., Kothe, E. J., He, J. L., Youssef, G. J., Enticott, P. G., ... \& Hyde, C. (2019). Motor imagery in children with DCD: A systematic and meta-analytic review of hand-rotation task performance. Neuroscience \& Biobehavioral Reviews.

Bek, J., Gowen, E., Vogt, S., Crawford, T. J., \& Poliakoff, E. (2019). Combined action observation and motor imagery influences hand movement amplitude in Parkinson's disease. Parkinsonism \& Related Disorders, 61, 126.

Bek, J., Holmes, P. S., Webb, J., Craig, C., Franklin, Z., Sullivan, M., . . Poliakoff, E. (2018). Home-based training to increase functional independence in Parkinson's through action observation and imagery. Archives of Physical Medicine and Rehabilitation, 99(10), e65 
Biotteau, M., Chaix, Y., \& Albaret J.M. (2016). What do we really know about motor learning in children with Developmental Coordination Disorder? Current Developmental Disorders Reports 3: 152-60.

Brookes, R. L., Nicolson, R. I., \& Fawcett, A. J. (2007). Prisms throw light on developmental disorders. Neuropsychologia, 45(8), 1921-1930.

Buccino, G., Molinaro, A., Ambrosi, C., Arisi, D., Mascaro, L., Pinardi, C., ... \& Galli, J. (2018). Action Observation Treatment Improves Upper Limb Motor Functions in Children with Cerebral Palsy: A Combined Clinical and Brain Imaging Study. Neural Plasticity, 2018.

Caligiore, D., Mustile, M., Spalletta, G., \& Baldassarre, G. (2017). Action observation and motor imagery for rehabilitation in Parkinson's disease: A systematic review and an integrative hypothesis. Neuroscience \& Biobehavioral Reviews, 72, 210-222.

Cantin, N., Polatajko, H. J., Thach, W. T., \& Jaglal, S. (2007). Developmental coordination disorder: exploration of a cerebellar hypothesis. Human Movement Science, 26(3), 491-509.

Causer, J., McCormick, S. A., \& Holmes, P. S. (2013). Congruency of gaze metrics in action, imagery and action observation. Frontiers in Human Neuroscience, 7, 604.

Cumming, J., \& Ramsey, R. (2009). Imagery interventions in sport. Advances in Applied Sport Psychology: A Review, 5-36.

Debrabant, J., Gheysen, F., Caeyenberghs, K., Van Waelvelde, H., \& Vingerhoets, G. (2013). Neural underpinnings of impaired predictive motor timing in children with Developmental Coordination Disorder. Research in Developmental Disabilities, 34(5), 1478-1487.

Deconinck, F. J. A., De Clercq, D., Savelsbergh, G. J., Van Coster, R., Oostra, A., Dewitte, G., \& Lenoir, M. (2006). Visual contribution to walking in children with developmental coordination disorder. Child: Care, Health and Development, 32(6), 711-722. 
Eaves, D. L., Riach, M., Holmes, P. S., \& Wright, D. J. (2016). Motor imagery during action observation: A brief review of evidence, theory and future research opportunities. Frontiers in Neuroscience, 10, 514. https://doi.org/10.3389/fnins.2016.00514.

Ertelt, D., \& Binkofski, F. (2012). Action observation as a tool for neurorehabilitation to moderate motor deficits and aphasia following stroke. Neural Regeneration Research, 7(26), 2063.

Ferguson, G. D., Wilson, P. H., \& Smits-Engelsman, B. C. M. (2015). The influence of task paradigm on motor imagery ability in children with developmental coordination disorder. Human Movement Science, 44, 81-90.

Fuchs, C. T., \& Caçola, P. (2018). Differences in accuracy and vividness of motor imagery in children with and without Developmental Coordination Disorder. Human movement Science, 60, 234-241.

Frank, C., Land, W. M., \& Schack, T. (2013). Mental representation and learning: The influence of practice on the development of mental representation structure in complex action. Psychology of Sport and Exercise, 14, 353-361. https://doi.org/10.1016/i.psychsport.2012.12.001

Guillot, A., Hoyek, N., Louis, M., \& Collet, C. (2012). Understanding the timing of motor imagery: recent findings and future directions. International Review of Sport and Exercise Psychology, 5(1), 3-22.

Hardwick, R. M., Caspers, S., Eickhoff, S. B., \& Swinnen, S. P. (2018). Neural correlates of action: Comparing meta-analyses of imagery, observation, and execution. Neuroscience and Biobehavioral Reviews, 94, 31-44.

Hayhoe, M., \& Ballard, D. (2005). Eye movements in natural behavior. Trends in Cognitive Sciences, 9(4), 188-194.

Henderson, S. E., Sugden, D. A. and Barnett, A. L. (2007). Movement assessment battery for children-2 second edition. London: The Psychological Corporation 
Heremans, E., Smits-Engelsman, B., Caeyenberghs, K., Vercruysse, S., Nieuwboer, A., Feys, P., \& Helsen, W. F. (2011). Keeping an eye on imagery: the role of eye movements during motor imagery training. Neuroscience, 195, 37-44.

Holmes, P. S., \& Collins, D. J. (2001). The PETTLEP approach to motor imagery: A functional equivalence model for sport psychologists. Journal of Applied Sport Psychology, 13(1), 60-83.

Hyde, C., Fuelscher, I., Williams, J., Lum, J. A., He, J., Barhoun, P., \& Enticott, P. G. (2018). Corticospinal excitability during motor imagery is reduced in young adults with developmental coordination disorder. Research in Developmental Disabilities, 72, 214-224.

Jeannerod, M. (2001). Neural simulation of action: A unifying mechanism for motor cognition. Neurolmage, 14, S103-S109. https://doi.org/10.1006/nimg.2001.0832

Kagerer, F. A., Bo, J., Contreras-Vidal, J. L., \& Clark, J. E. (2004). Visuomotor adaptation in children with developmental coordination disorder. Motor Control, 8(4), 450-460.

Kagerer, F. A., Contreras-Vidal, J. L., Bo, J., \& Clark, J. E. (2006). Abrupt, but not gradual visuomotor distortion facilitates adaptation in children with developmental coordination disorder. Human Movement Science, 25(4-5), 622-633.

Kawato, M. (1999). Internal models for motor control and trajectory planning. Current Opinion in Neurobiology, 9(6), 718-727.

Kilteni, K., Andersson, B. J., Houborg, C., \& Ehrsson, H. H. (2018). Motor imagery involves predicting the sensory consequences of the imagined movement. Nature Communications, 9(1), 1617.

Kim, T., Frank, C., \& Schack, T. (2017). A systematic investigation of the effect of action observation training and motor imagery training on the development of mental representation structure and skill performance. Frontiers in Human Neuroscience, 11, 499. https://doi.org/10.3389/fnhum.2017.00499. 
King, B. R., Kagerer, F. A., Harring, J. R., Contreras-Vidal, J. L., \& Clark, J. E. (2011). Multisensory adaptation of spatial-to-motor transformations in children with developmental coordination disorder. Experimental Brain Research, 212(2), 257-265.

Krakauer, J. W. (2009). Motor learning and consolidation: the case of visuomotor rotation. In Progress in motor control (pp. 405-421). Springer, Boston, MA.

Land, M. F. (2009). Vision, eye movements and natural behavior. Visual Neuroscience, 26(1), 51-62. DOI: https://doi.org/10.1017/\$0952523808080899

Lei, Y., Bao, S. and Wang, J. (2016). The combined effects of action observation and passive proprioceptive training on adaptive motor learning. Neuroscience, 331, 91-98. DOI: https://doi.org/10.1016/i.neuroscience.2016.06.011

Marshall, B., Wright, D.J., Holmes, P.S., \& Wood, G. (2019). Combining action observation and motor imagery improves eye-hand coordination during novel visuomotor task performance. Journal of Motor Behavior, 1-8

Miles, C. A. L., Wood, G., Vine, S. J., Vickers, J. N., \& Wilson, M. R. (2015). Quiet eye training facilitates visuomotor coordination in children with developmental coordination disorder. Research in Developmental Disabilities, 40, 31-41.

Morgan, R., \& Long, T. (2012). The effectiveness of occupational therapy for children with developmental coordination disorder: A review of the qualitative literature. British Journal of Occupational Therapy, 75, 10-18. doi:10.4276/030802212×13261082051337

Ong, N. T. and Hodges, N. J. (2010). Absence of after-effects for observers after watching a visuomotor adaptation. Experimental Brain Research, 205(3), 325-334.

Ong, N. T., Larssen, B. C. and Hodges, N. J. (2012). In the absence of physical practice, observation and imagery do not result in updating of internal models for aiming. Experimental Brain Research, 218(1), 9-19.

Parr, J. V. V., Vine, S. J., Harrison, N. R., \& Wood, G. (2018). Examining the spatiotemporal disruption to gaze when using a myoelectric prosthetic hand. Journal of Motor Behavior, 50(4), 416-425. 
801

802

803

804

805

806

807

808

809

810

811

812

813

814

815

816

817

818

819

820

821

822

823

824

825

826

827

828

Parr, J. V. V., Vine, S. J., Wilson, M. R., Harrison, N. R., \& Wood, G. (2019). Visual attention, EEG alpha power and T7-Fz connectivity are implicated in prosthetic hand control and can be optimized through gaze training. Journal of Neuroengineering and Rehabilitation, 16(1), 52.

Romano-Smith, S., Wood, G., Wright, D. J., \& Wakefield, C. J. (2018). Simultaneous and alternate action observation and motor imagery combinations improve aiming performance. Psychology of Sport and Exercise, 38, 100-106. https://doi.org/10.1016/i.psychsport.2018.06.003

Romano Smith, S., Wood, G., Coyles, G., Roberts, J. W., \& Wakefield, C. J. (2019). The effect of action observation and motor imagery combinations on upper limb kinematics and EMG during dart-throwing. Scandinavian Journal of Medicine \& Science in Sports.

Sailer, U., Flanagan, J. R., \& Johansson, R. S. (2005). Eye-hand coordination during learning of a novel visuomotor task. Journal of Neuroscience, 25(39), 8833-8842.

Schack, T., \& Mechsner, F. (2006). Representation of motor skills in human long-term memory. Neuroscience Letters, 391, 77-81. https://doi.org/10.1016/i.neulet.2005.10.009

Scott, M. W., Emerson, J. R., Dixon, J., Tayler, M. A., \& Eaves, D. L. (2019). Motor imagery during action observation enhances automatic imitation in children with and without developmental coordination disorder. Journal of Experimental Child Psychology, 183, 242-260.

Słowiński, P., Baldemir, H., Wood, G., Alizadehkhaiyat, O., Coyles, G., Vine, S., ... \& Wilson, M. (2019). Gaze training supports self-organization of movement coordination in children with developmental coordination disorder. Scientific Reports, 9(1), 1712.

Teulings, H. L., Contreras-Vidal, J. L., Stelmach, G. E., \& Adler, C. H. (1997). Parkinsonism reduces coordination of fingers, wrist, and arm in fine motor control. Experimental Neurology, 146(1), 159-170.

Vogt, S., Di Rienzo, F., Collet, C., Collins, A., \& Guillot, A. (2013). Multiple roles of motor imagery during action observation. Frontiers in Human Neuroscience, 7, 807. 
Wang, J., \& Lei, Y. (2015). Direct-effects and after-effects of visuomotor adaptation with one arm on subsequent performance with the other arm. Journal of Neurophysiology, 114(1), 468-473.

Wilson, B. N., Kaplan, B. J., Crawford, S. G. and Roberts, G. (2007). The developmental coordination disorder questionnaire 2007 (DCDQ'07). Alberta Children's Hospital, Calgary, Canada.

Wilson, P.H., Adams, I. L., Caeyenberghs, K., Thomas, P., Smits-Engelsman, B., \& Steenbergen, B. (2016). Motor imagery training enhances motor skill in children with DCD: A replication study. Research in Developmental Disabilities, 57, 54-62.

Wilson, P.H., \& Butson, M., (2007). Deficits underlying DCD. In: Geuze, R.H. (Ed.), Developmental Coordination Disorder: A Review of Current Approaches. Solal Editeurs, Marseille, pp. 115-119 (Chapter 4).

Wilson, P. H., Ruddock, S., Smits-Engelsman, B., Polatajko, H. and Blank, R. (2013). Understanding performance deficits in developmental coordination disorder: a meta-analysis of recent research. Developmental Medicine and Child Neurology, 55(3), 217-228. DOI: https://doi.org/10.1111/j.1469-8749.2012.04436.x

Wilson, P.H., Thomas, P. R. and Maruff, P. (2002). Motor imagery training ameliorates motor clumsiness in children. Journal of Child Neurology, 17(7), 491-498. DOI: https://doi.org/10.1177/088307380201700704

Wilson, M., McGrath, J., Vine, S., Brewer, J., Defriend, D. and Masters, R. (2010). PsychoMotor Control in a virtual laparoscopic surgery training environment: gaze control parameters differentiate novices from experts. Surgical Endoscopy, 24(10), 2458-2464.

Wolpert, D. M. (1997). Computational approaches to motor control. Trends in Cognitive Sciences, 1(6), 209-216.

Wood, G., Miles, C. A., Coyles, G., Alizadehkhaiyat, O., Vine, S. J., Vickers, J. N., \& Wilson, M. R. (2017). A randomized controlled trial of a group-based gaze training intervention for children with Developmental Coordination Disorder. PloS One, 12(2), e0171782. 
857

858

859

860

861

862

863

864

865

866

867

Wright, D. J., Williams, J., \& Holmes, P. S. (2014). Combined action observation and imagery facilitates corticospinal excitability. Frontiers in Human Neuroscience, 8, 951.

Wright, D. J., Wood, G., Eaves, D. L., Bruton, A. M., Frank, C., \& Franklin, Z. C. (2018). Corticospinal excitability is facilitated by combined action observation and motor imagery of a basketball free throw. Psychology of Sport and Exercise, 39, 114-121.

Zimmermann-Schlatter, A., Schuster, C., Puhan, M. A., Siekierka, E., \& Steurer, J. (2008). Efficacy of motor imagery in post-stroke rehabilitation: a systematic review. Journal of Neuroengineering and Rehabilitation, 5(1), 8.

Zwicker, J. G., Missiuna, C., Harris, S. R., \& Boyd, L. A. (2010). Brain activation of children with developmental coordination disorder is different than peers. Pediatrics, 126(3), e678-e686. 